\title{
Impacto do déficit de investimentos para o tratamento da doença de chagas no Brasil: revisão narrativa
}

RESUMO | Objetivo: discutir sobre o impacto do déficit de investimentos para o tratamento da Doença de Chagas no Brasil. Método: trata-se de uma revisão narrativa da literatura realizada nas seguintes bases de dados: SCIELO, LILACS, BIREME e MEDLINE. A amostra final foi composta por 27 artigos científicos publicados entre o período de 2000 a 2020. Resultados: o Brasil é um dos países com maior prevalência de pacientes portadores da doença, porém pouco se avançou em pesquisas nessa área, de modo a repercutir em um baixo incentivo e investimento dos governantes e da indústria farmacêutica para a Doença de Chagas, tendo em vista a doença ser de progressão lenta e o diagnóstico e o tratamento serem tardios. Conclusão: fica evidente a falta de investimento e políticas públicas que possibilitem o diagnóstico e o tratamento precoce da doença tendo como conseqüência um déficit na qualidade de vida dos pacientes.

Palavras-chaves: Doença de Chagas; Investimentos em Saúde; Terapêutica.

ABSTRACT | Objective: to discuss the impact of the investment deficit for the treatment of Chagas Disease in Brazil. Method: This is a narrative review of the literature conducted in the following databases: SCIELO, LILACS, BIREME and MEDLINE. The final sample consisted of 27 scientific articles published between 2000 and 2020. Results: Brazil is one of the countries with the highest prevalence of patients with the disease, but little progress has been made in research in this area, so as to have a low incentive and investment from governments and the pharmaceutical industry for Chagas Disease, in view of the disease being of slow progression and the diagnosis and treatment are late. Conclusion: it is evident the lack of investment and public policies that make possible the diagnosis and early treatment of the disease with the consequence of a deficit in the quality of life of the patients.

Keywords: Chagas disease; Investments; Therapeutics.

RESUMEN | Objetivo: discutir el impacto del déficit de inversión para el tratamiento de la enfermedad de Chagas en Brasil. Método: se trata de una revisión narrativa de la literatura realizada en las siguientes bases de datos: SCIELO, LILACS, BIREME y MEDLINE. La muestra final consistió en 27 artículos científicos publicados entre 2000 y 2020. Resultados: el Brasil es uno de los países con mayor prevalencia de pacientes con la enfermedad, pero se ha avanzado poco en la investigación en esta área, para tener un bajo incentivo e inversión de los gobiernos y de la industria farmacéutica para la enfermedad de Chagas, considerando que la enfermedad es de progresión lenta y el diagnóstico y tratamiento son tardíos. Conclusión: es evidente la falta de inversión y de políticas públicas que permitan el diagnóstico y tratamiento precoz de la enfermedad con el consiguiente déficit en la calidad de vida de los pacientes.

Palabras claves: Enfermedad de Chagas; Inversiones en Salud; Terapéutica.

\section{Patrick Leonardo Nogueira da Silva}

Enfermeiro, Mestrando pelo Programa de Pós-Graduação em Cuidado Primário em Saúde da Universidade Estadual de Montes Claros (PPGCPS/UNIMONTES), Professor da Escola Técnica de Saúde do Centro de Educação Profissional e Tecnológica (ETS/CEPT) da UNIMONTES. Montes Claros, MG, Brasil.

ORCID: 0000-0003-2399-9526

\section{Fabrícia Josely Oliveira Barbosa}

Enfermeira, Mestre em Gestão de Serviços de Saúde pela Universidade Federal de Minas Gerais (PPGGSS/UFMG). Belo Horizonte, MG, Brasil.

ORCID: 0000-0003-0786-0284

\section{Vitor Hugo Maraslis Soares}

Acadêmico do curso de graduação em Medicina da Universidade Vale do Rio Doce (UNIVALE). Governador Valadares, MG, Brasil. ORCID: 0000-0001-8518-5582

\section{Fábio Batista Miranda}

Enfermeiro, Doutorando pelo Programa de Pós-Graduação em Enfermagem e Biociências da Universidade Federal do Estado do Rio de Janeiro (PPGENFBIO/UNIRIO). Rio de Janeiro, RJ, Brasil.

ORCID: 0000-0003-0934-980X

\section{Ana Patrícia Fonseca Coelho Galvão}

Enfermeira, Doutoranda em Ciências da Saúde pela Faculdade de Ciências Médicas da Santa Casa de São Paulo (FCMSCSP), Professora do Departamento de Enfermagem da Universidade Ceuma (UNICEUMA). São Luís, MA, Brasil. ORCID: 0000-0003-3376-5678

\section{Carolina dos Reis Alves}

Enfermeira, Doutora em Ciências da Saúde, Professora do Departamento de Enfermagem da Faculdade Santo Agostinho (FASA). Montes Claros, MG, Brasil.

ORCID: 0000-0003-2107-6306
Recebido em: 21/01/2021

Aprovado em: 29/01/2021

INTRODUÇÃO

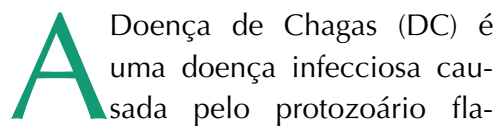
gelado Trypanosoma cruzi (T. cruzi), popularmente conhecida como doença do coração crescido, e constitui um sério problema de saúde pública, além de um dos mais importantes problemas médico-social a qual desenvolve uma serie de particularidades e conseqüências para os seres humanos ${ }^{(1,2)}$. A DC humana é transmitida pelas fezes do vetor, sendo este o Triatoma infestans (T. infestans), mais conhecido como barbeiro, quando o hospedeiro entra em contato, além 
disso, é transmitido por via oral, através da ingestão de alimentos contaminados, hemotransfusão ou durante o período gravídico, ou seja, da mãe infectada ao feto. A infecção chagásica caracteriza-se por duas fases, sendo a primeira a fase aguda que pode ser evidente ou não, e a segunda a fase crônica que acompanha o indivíduo por toda a vida, sendo considerada pela Organização Mundial da Saúde (OMS) uma afecção negligenciada ${ }^{(3,4)}$.

A DC é de natureza enzoótica, encontrando-se presente nas Américas há milhares de $\operatorname{anos}^{(5,6)}$. Nos anos 40, em decorrência a uma série de fatores bioecológicos e político-sociais, gerados pela migração de pessoas do meio rural para os grandes centros urbanos (êxodo rural), o homem se torna um reservatório da DC, o que gera um sério problema de saúde pública, desmistificando a idéia de ser uma doença rural ${ }^{(5,7)}$. A descoberta e descrição da DC humana no Brasil se deram em 1909, no interior de Minas Gerais, por Ribeiro Justiniano das Chagas, pesquisador do Instituto Oswaldo Cruz (FIOCRUZ), sendo uma das mais bem sucedidas descobertas da biologia, porém de lá até os dias atuais pouco se avançou na evolução de diagnósticos e tratamentos dessa doença. Acredita-se que o motivo desse desinteresse em novas pesquisas é o fato de essa ser uma doença que acomete principalmente a população de baixa renda, que apresenta poucos recursos para o custeio de seus tratamentos. A DC tem uma maior distribuição no continente americano, sendo que no Brasil chegou a atingir cerca de $40 \%$ do território nacional e os Estados com mais índices de casos são: Minas Gerais, Goiás, Bahia, São Paulo, Acre, Amazonas e Amapá(8).

O diagnóstico da DC se dá, em sua maioria das vezes, por meio de testes de sangue na fase aguda, e na fase crônica são realizados testes enzimáticos específicos, tais como o teste de Enzyme-Linked Immunosorbent Assay (ELISA) ${ }^{(4)}$. Segundo informações do Projeto de Controle de Doenças Prioritárias (PCDP) do National Institutes of Health (NIH) e do Banco
Mundial (BM), existe uma prevalência de oito a nove milhões de pessoas infectadas, tendo no Brasil, provavelmente, de dois a três milhões de portadores ${ }^{(9,10)}$. $\mathrm{O}$ tratamento da DC segue o mesmo há mais de 50 anos, usando hoje apenas um medicamento, o benznidazol, uma vez que o uso do nifurtimox foi suspenso devido efeitos colaterais negativos, valendo considerar que estudos mostraram baixa eficiência dessa medicação no tratamento do paciente na fase crônica, sendo um tratamento limitado, não apresentando

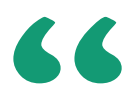

evolução nas pesquisas por parte da indústria farmacêutica para a produção de um novo quimioterápico desde a descoberta da doença ${ }^{(11,12)}$.

Ao analisar a bibliografia consultada para o estudo, percebe-se que pouco ou nada se discorre sobre o melhoramento no tratamento da DC, evidência negligenciada sobre essa doença, sendo caracterizada como "doença de pobre" a qual tem os seus maiores índices epidemiológicos nos países subdesenvolvidos ${ }^{(13)}$. Ainda, salienta-se que a falta de incentivo por parte dos governantes afetam na qualidade de vida (QV) dos portadores da DC, bem como dificulta o trabalhado dos médicos, uma vez que a realidade econômica dos seus pacientes não condiz com os altos custos das medicações durante o tratamento ${ }^{(14)}$.

Sendo assim, este estudo objetivou discutir sobre o impacto do déficit de investimentos para o tratamento da DC no Brasil por meio de uma revisão narrativa.

\section{MÉTODO}

Trata-se de um estudo descritivo, exploratório, documental, retrospectivo, com base em uma revisão narrativa da literatura. A busca pelos artigos foi realizada por meio das seguintes bases de dados online: Scientific Eletronic Library Online (SciELO), Literatura Latino-Americana e do Caribe em Ciências da Saúde (LILACS), Biblioteca Regional de Medicina (BIREME) e Medical Literature Analysis and Retrieval System Online (MEDLINE). A consulta a estes bancos de dados foi tida como critério inicial para seleção. Optou-se por estas bases de dados por serem as mesmas uma das principais fontes de publicações científicas na atualidade e, a partir de seu sistema de busca, foram utilizadas as seguintes palavras-chave: "DC"; "Investimentos em Saúde"; e "Terapêutica".

Foram adotados os seguintes critérios de inclusão para a captação dos artigos: estar indexado nas respectivas bases de dados; ter o resumo disponível para leitu- 
Tabela 1 - Perfil da amostragem conforme busca nas bases de dados da SCIELO, LILACS, BIREME e MEDLINE.

\begin{tabular}{|c|c|c|c|c|c|c|c|c|c|c|}
\hline \multirow{2}{*}{ Descritores } & \multicolumn{2}{|c|}{ SCIELO } & \multicolumn{2}{|c|}{ LILACS } & \multicolumn{2}{|c|}{ BIREME } & \multicolumn{2}{|c|}{ MEDLINE } & \multicolumn{2}{|c|}{ TOTAL } \\
\hline & $\mathbf{n}$ & $\%$ & $\mathbf{n}$ & $\%$ & n & $\%$ & $\mathrm{n}$ & $\%$ & $\mathrm{n}$ & $\%$ \\
\hline $\begin{array}{l}\text { "DC" AND "Investimen- } \\
\text { tos em saúde" }\end{array}$ & 00 & 0,0 & 00 & 0,0 & 00 & 0,0 & 01 & 0,1 & 01 & 0,1 \\
\hline "DC" AND "Terapêutica" & 09 & 0,6 & 114 & 6,2 & 00 & 0,0 & 1563 & 85,9 & 1686 & 92,7 \\
\hline $\begin{array}{l}\text { Investimentos em saúde } \\
\text { AND Terapêutica }\end{array}$ & 00 & 0,0 & 02 & 0,1 & 00 & 0,0 & 129 & 7,1 & 131 & 7,2 \\
\hline $\begin{array}{l}\text { "DC" AND "Investi- } \\
\text { mentos em saúde" AND } \\
\text { "Terapêutica" }\end{array}$ & 00 & 0,0 & 00 & 0,0 & 00 & 0,0 & 00 & 0,0 & 00 & 0,0 \\
\hline Total & 09 & 0,6 & 116 & 6,3 & 00 & 0,0 & 1693 & 93,1 & 1818 & 100,0 \\
\hline
\end{tabular}

Fonte: Autoria própria, 2020.

Tabela 2 - Perfil da amostra após aplicação dos critérios de elegibilidade.

\section{Descritores}

\section{SCIELO LILACS BIREME MEDLINE TOTAL}

\begin{tabular}{lcccccccccc} 
& AS & AU & AS & AU & AS & AU & AS & AU & AS & AU \\
$\begin{array}{l}\text { "DC" AND "Investimentos } \\
\text { em saúde" }\end{array}$ & 00 & 00 & 00 & 00 & 00 & 00 & 01 & 01 & 01 & 01 \\
\hline "DC" AND "Terapêutica" & 09 & 06 & 114 & 10 & 00 & 00 & 1563 & 05 & 1686 & 21 \\
$\begin{array}{l}\text { Investimentos em saúde } \\
\text { AND Terapêutica }\end{array}$ & 00 & 00 & 02 & 00 & 00 & 00 & 129 & 05 & 131 & 05 \\
$\begin{array}{l}\text { "DC" AND "Investimentos em } \\
\text { saúde" AND "Terapêutica" }\end{array}$ & 00 & 00 & 00 & 00 & 00 & 00 & 00 & 00 & 00 & 00 \\
Total & 09 & 06 & 116 & 10 & 00 & 00 & 1693 & 11 & 1818 & 27
\end{tabular}

Fonte: Autoria própria, 2020. AS = Artigos Selecionados (amostragem). AU = Artigos Utilizados (amostra). ra na língua portuguesa; estar disponível gratuitamente para download; ser artigo nacional ou internacional; e estar publicado entre o período de janeiro de 2000 a dezembro de 2020. A busca resultou em uma amostragem de 1818 artigos (Tabela 1), sendo que, após a aplicação dos critérios de elegibilidade do estudo, a amostra final foi composta por 27 artigos publicados entre os anos de 2000 a 2020 (Tabela 2). Ainda, foi realizada uma leitura cuidadosa de todos os artigos selecionados a fim de garantir a aplicação dos critérios de elegibilidade. A coleta de dados ocorreu no $1^{\circ}$ semestre de 2020, entre os meses de abril a junho do respectivo ano, pelo pesquisador responsável. Para o mesmo, foi utilizado um formulário estruturado como instrumento de coleta de dados.

\section{RESULTADOS}

A amostra deste estudo, após seleção nas bases de dados (SCIELO, LILACS, BIREME e MEDLINE) e aplicação dos critérios de elegibilidade, foi representada em uma tabela conforme as seguintes variáveis: título, autor, ano de publicação, periódico, objetivo e método (Tabela 3).

Tabela 3 - Apresentação da amostra do estudo, segundo o título, autor, ano, periódico, objetivo e método, após seleção nas bases de dados (SCIELO, LILACS, BIREME e MEDLINE) e aplicação dos critérios de elegibilidade. ( $n=27)$

\begin{tabular}{|c|c|c|c|c|c|c|}
\hline $\mathrm{N}^{\circ}$ & Título & Autor & Ano & Periódico & Objetivo & Método \\
\hline 1 & $\begin{array}{l}\text { Utilidade das estratégias } \\
\text { de PCR para diagnóstico } \\
\text { precoce da reativação } \\
\text { da doença de Chagas } \\
\text { e acompanhamento do } \\
\text { tratamento no transplante } \\
\text { cardíaco }\end{array}$ & Diez et al.(2) & 2007 & $\begin{array}{l}\text { Am. J. Trans- } \\
\text { plant. }\end{array}$ & $\begin{array}{l}\text { Avaliar a incidência de reativação em } \\
\text { uma série de pacientes submetidos a } \\
\text { transplante cardíaco para o estágio } \\
\text { final da cardiomiopatia chagásica. }\end{array}$ & $\begin{array}{l}\text { Estudo ecológico realizado por } \\
\text { meio de testes de reativação } \\
\text { diagnóstica para a DC. }\end{array}$ \\
\hline 2 & $\begin{array}{l}\text { Il Consenso Brasileiro em } \\
\text { Doença de Chagas, } 2015\end{array}$ & Dias et al..$^{(3)}$ & 2016 & $\begin{array}{l}\text { Epidemiol. Serv. } \\
\text { Saúde }\end{array}$ & $\begin{array}{l}\text { Sistematizar estratégias de diagnósti- } \\
\text { co, tratamento, prevenção e controle } \\
\text { da DC no país, de modo a refletir as } \\
\text { evidências científicas disponíveis. }\end{array}$ & $\begin{array}{l}\text { Fundamentou-se na articula- } \\
\text { ção e contribuição estratégica } \\
\text { de especialistas brasileiros } \\
\text { com conhecimento, expe- } \\
\text { riência e atualização sobre } \\
\text { diferentes aspectos da DC. }\end{array}$ \\
\hline 3 & $\begin{array}{c}\text { Métodos de diagnóstico } \\
\text { para a Doença de Chagas: } \\
\text { uma atualização }\end{array}$ & $\begin{array}{c}\text { Alves et } \\
\text { al. }^{(4)}\end{array}$ & 2018 & $\begin{array}{l}\text { Rev. Bras. An. } \\
\text { Clin. }\end{array}$ & $\begin{array}{l}\text { Realizar uma atualização sobre os } \\
\text { métodos de diagnóstico para a DC. }\end{array}$ & $\begin{array}{l}\text { Utilizaram-se guias, as bases } \\
\text { de dados SCIELO, LILACS, } \\
\text { e como descritor, DC, cujos } \\
\text { artigos foram publicados entre } \\
\text { os anos de } 1997 \text { e } 2017 \text {. }\end{array}$ \\
\hline
\end{tabular}


Análise da soroprevalência da Doença de Chagas em

4 uma cidade do norte de Minas Gerais durante o período de 2012 a 2014

\section{Brasil et al (5) $2014 \quad$ Rev. Univ. Vale Rio Verde}

Analisar a soroprevalência da DC em uma cidade do norte de Minas Gerais durante o período de 2012 a 2014
Estudo exploratório-descritivo, documental, com abordagem quantitativa, sendo uma investigação retrospectiva dos casos confirmados da DC durante janeiro/2012 a janeiro/2014.

Estimar a influência das modificações ambientais de origem antrópica, objetivando melhor compreensão da situação atual e da eficácia das medidas de controle dos triatomíneos.

Estudo biogeográfico Pública

Analisar dois períodos da trajetória de consolidação e legitimação científica e social da tripanossomíase americana

Estudo histórico construção de um fato

6 científico e de um problema de saúde pública no Brasil

Schofield; Jannin; Salvatella(9)
Kropf; Azevedo;

Ferreira $^{(8)}$
2000 Colet. ou DC.

0 futuro do controle da
doença de Chagas

Revisão sistemática ma revisão crítica sobre

8 a quimioterapia da doença de Chagas

$\begin{array}{ccc}\text { Coura; } & 2002 & \begin{array}{c}\text { Mem. Inst. } \\ \text { Oswaldo Cruz }\end{array}\end{array}$

Fazer uma introdução histórica dos fármacos ensaiados contra a DC a partir de 1912 com os trabalhos de Mayer e Rocha Lima até o uso experimental da nitrofurazona.

\begin{tabular}{|c|c|c|c|c|}
\hline 9 & $\begin{array}{l}\text { Nanomedicamentos con- } \\
\text { tra a doença de Chagas: } \\
\text { uma atualização sobre } \\
\text { terapêutica, profilaxia e } \\
\text { diagnóstico }\end{array}$ & $\begin{array}{c}\text { Morilla; } \\
\text { Romero }^{(12)}\end{array}$ & 2015 & $\begin{array}{l}\text { Nanomed. } \\
\text { (Lond) }\end{array}$ \\
\hline
\end{tabular}

Tecnologias que empre-

10 gam fármacos antiparasitários para tratamento da doença Chagas mun. Inf. Inov. Saúde
Discutir sobre a terapêutica, profilaxia e diagnóstico por meio de nanomedicamentos contra a DC.
Revisão crítica
Apresentar um mapeamento tecnológico dos documentos de patentes relacionadas a tecnologias que empregam fármacos antiparasitários para tratamento da DC, por intermédio do banco de patentes do United States Patent and Trademark Office - USPTO.
Revisão sistemática

Sumarizar e discutir os dados da

Fundação Nacional de Saúde (FNS) sobre o estado atual dos vetores da DC

Estudo histórico

Estudo exploratório, documental, retrospectivo, com base em uma revisão sistemática de patentes relacionadas a tecnologias que empregam fármacos antiparasitários o tratamento da DC.

11 Doença de Chagas no Vinhaes; Brasil $\operatorname{Dias}^{(15)}$

2000

Cad. Saúde
Pública no Brasil.

Epidemiologia, controle

12 e vigilância da doença de Chagas - 100 anos após sua descoberta

Pesquisas prioritárias

13

sobre doença de Chagas

na Amazônia: agenda de curto-médio prazo
Dias et al. $^{(17)} 2001 \quad$ Rev. Soc. Bras.
Med. Trop.
Discutir sobre a epidemiologia, controle e vigilância da DC em 100 anos após sua descoberta.
Revisão sistemática Oswaldo Cruz
Relatar sobre a agenda de curto-médio prazo das pesquisas prioritárias com relação à DC na Amazônia
Relatório técnico
Aplicando uma lente de equidade à saúde e mor-

talidade infantil: mais do

mesmo não é suficiente

Victória et
al. $^{(18)}$ $2003 \quad$ Lancet

Informar sobre as lacunas na mortalidade infantil entre países ricos e pobres.
Revisão sistemática 
Mudanças no paradigma da conduta clínica e

15 terapêutica da doença de Chagas: avanços e perspectivas na busca da integralidade da saúde

16 Doença de Chagas: uma atualização bibliográfica

Terapia celular na doença
de Chagas Doença de Chagas: passa-
do, presente e futuro

Doença de Chagas aguda

19 no Brasil: série histórica de 2000 a 2013

A imunização genética induz respostas imunológicas protetoras específicas

20 para antígenos e diminui a gravidade da doença na infecção por Trypanosoma cruzi
A imunidade tipo 1 fornece proteção mucosa

21 e sistêmica ideal contra um patógeno intracelular invasivo da mucosa $\begin{array}{cc}\text { Dias et al. }{ }^{(19)} 2016 \quad \begin{array}{c}\text { Epidemiol. Serv. } \\ \text { Saúde }\end{array} & \text { dit }\end{array}$ na busca da integralidade da saúde

tendo em vista as mudanças no para-

digma da conduta clínica e terapêutica

da DC.
Opinar sobre os avanços e perspectivas
Estudo de opinião

Rever as vias de transmissibilidade,

bem como as formas diagnósticas, para a DC.
Revisão bibliográfica ra; Lima ${ }^{(20)} 2019$ Clín.
Discutir sobre a terapia celular na DC à luz da literatura científica.
Revisão sistemática
Abordar sobre o contexto histórico da Colet.
Editorial

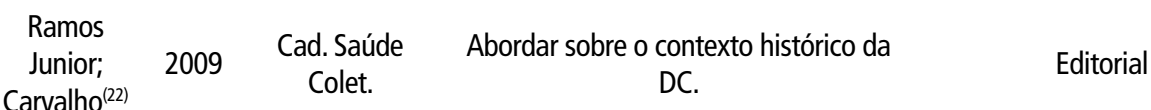
Carvalho ${ }^{(22)}$
Descrever a DC aguda no Brasil por

Brasil(23) 2015 Bol. Epidemiol. meio de uma série histórica de 2000 a 2013.
Revisão sistemática $\underset{\text { ton }^{(24)}}{\text { Garg; Tarle- }} 2002$ Infect. Immun.
Avaliar a eficácia dos genes ASP-1, ASP-2 e TSA-1 da família trans-sialidase (ts) do T. cruzi como vacinas genéticas.
Estudo ecológico

\section{Hoft; \\ Eickhoff(25) 2005 Infect. Immun. quanto ao fornecimento de proteção mucosa e sistêmica ideal contra um patógeno intracelular invasivo da mucosa.}

Investigar sobre a imunidade tipo 1
Estudo ecológico
A doença de Chagas está associada a um desfecho

22 ruim no acompanhamento de 1 ano após a terapia de ressincronização cardíaca
Anticoagulação nas manifestações cardíacas

23 da doença de Chagas e acidente vascular cerebral isquêmico cardioembólico
Monteiro et al. (27)

2018

$\begin{array}{ccc}\begin{array}{c}\text { Passos et } \\ \text { al. }{ }^{(26)}\end{array} & 2019 & \text { Rev. Assoc. } \\ & & \text { Med. Bras. }\end{array}$
Avaliar o prognóstico após terapia de ressincronização cardíaca (TRC) em uma população em que a DC é uma causa freqüente de insuficiência cardíaca (IC).
Coorte retrospectiva realizada entre janeiro/2015 e dezembro/2016
Descrever as características da anticoagulação em pacientes com manifestações cardíacas da DC e comparar os participantes com e sem acidente vascular cerebral isquêmico cardioembólico (AVCIC)
Estudo de coorte retrospectivo com abordagem quantitativa
Investigação clínica e ma-

24
Avaliar os achados da manometria anal (principalmente o Reflexo Inibitório Retoanal) em pacientes chagásicos com constipação crônica, com e sem megacólon, e correlacionar esses achados com dados clínicos e demográficos.

\section{Analisar a segurança do Ecocardio-} grama sob Estresse com Dobutamina-Atropina (EED) em uma população de chagásicos com suspeita clínica de coronariopatia. 


\author{
Doença de Chagas: 0 que \\ 26

\begin{abstract}
é conhecido e o que deve
ser melhorado: uma visão sistêmica
\end{abstract}

\begin{tabular}{|c|c|}
\hline $\begin{array}{l}\text { Coura; } \\
\text { Borges-Pe- } \\
\text { reira }^{(30)}\end{array}$ & 2012 \\
\hline
\end{tabular}

Realizar uma ampla revisão sobre 0 que deve ser melhorado no conhecimento da $D C$, não somente através da análise dos principais trabalhos publicados sobre os tópicos discutidos, mas em grande parte com base na experiência sobre 0 assunto, dos últimos 50 anos (1961-2011).

\begin{tabular}{|c|c|c|c|c|c|c|}
\hline 27 & $\begin{array}{l}\text { Acometimento cardíaco } \\
\text { em Casos de Doença } \\
\text { de Chagas Aguda da } \\
\text { Amazônia }\end{array}$ & $\begin{array}{l}\text { Barbosa- } \\
\text {-Ferreira et } \\
\text { al. }{ }^{(31)}\end{array}$ & 2010 & $\begin{array}{c}\text { Arq. Bras. } \\
\text { Cardiol. }\end{array}$ & $\begin{array}{l}\text { Descrever o acometimento cardíaco em } \\
\text { cinco pacientes autóctones da Amazô- } \\
\text { nia com diagnóstico de DC aguda. }\end{array}$ & Relato de casos \\
\hline
\end{tabular}

Fonte: Autoria própria, 2020.

\section{DISCUSSÃO}

Características epidemiológicas da DC humana

Os T. infestans são animais silvestres, sendo o seu habitat natural as florestas, porém devido às ações antrópicas representadas pelos desmatamentos e ao forte movimento de urbanização, o homem passou a fazer parte do ciclo epidemiológico da doença, tornando-se um transmissor ${ }^{(5,15)}$. A DC caracteriza-se por atingir uma população economicamente vulnerável, com acesso precário aos serviços de saúde, as ações de promoção de saúde e prevenção de doenças, estando essa mais favorável ao adoecimento devido às precárias condições de moradias, a uma maior presença de agravos e exposição a outras doenças ${ }^{(5,15-18)}$.

O nível de transmissão nos domicílios foi considerado insignificante no Brasil, o qual the conferiu um certificado pela Comissão Intergovernamental do Cone Sul, afirmando que esse não seria mais um meio de contaminação no país, mas vale ressaltar que existem diferentes espécies de triatomíneos silvestres, onde ficou evidente a sua capacidade de adaptação nos domicílios na região nordeste do país ${ }^{(19)}$. A transmissão da doença se dá de diversas maneiras: transmissão vetorial, o inseto contaminando após picar o homem evacua no local e o indivíduo acaba coçando a região atingida lançando, dessa maneira, o protozoário na corrente sanguínea; a transmissão oral, o indivíduo ingere o alimento contaminado com o protozoário; a transmissão vertical (congênita), a qual a mãe transmite para o filho; a transmissão por hemotransfusão e doação e transplante de órgãos e tecidos (DTOT); e a transmissão acidental, que acontece por um erro na captura do barbeiro, aleitamento materno, um acidente de trabalho com agulhas contaminadas, dentre outros ${ }^{(3)}$.

Para anos mais recentes (Sistema de Informação de Agravos e Notificações [SINAN], 2000 a 2013), com a limitação operacional já indicada previamente, verificou-se que a forma de transmissão oral foi a mais freqüente em todos os anos $(n=1.081,68,9 \%)$, seguida pela transmissão vetorial em 100 casos $(6,4 \%)^{(3,19)}$. Sendo assim, a única maneira de controlar essa transmissão é por meio de produtos químicos usados pelos agentes de endemias da vigilância epidemiológica diretamente nos domicílios, poleiros, paióis e demais estruturas físicas próximas as residências"(3). "A DC é dividida em duas fases: a crônica (tardia, de evolução lenta e com baixa parasitemia) e a aguda (inicial, de rápida duração, com elevada parasitemia e geralmente auto-limitada) $)^{\prime \prime 20: 104)}$. O seu diagnóstico é realizado diferentemente nas duas fases. Na faze aguda é feito através de exames de sangue a fresco, gota espessa e esfregaço, podendo o indivíduo apresentar ou não sintomas aparentes, caso o profissional detenha uma suspeita clara sobre a doença e os testes apresentarem negati- vos, ele deve solicitar um teste de concentração (microhematócrito ou Strout) cuja eficiência pode chega a $90 \%$. Na fase crônica, onde já se tem uma baixa na parasitemia, o diagnostico deve ser feito exclusivamente por meio da sorologia "(ELISA com antígeno total ou frações semi-purificadas do parasito ou a IFI) e outro de alta especificidade (ELISA, utilizando antígenos recombinantes específicos do T. cruzi $)^{\prime \prime(20: 104)}$.

Dificuldades encontradas para a realização do tratamento da DC no Brasil

Os países da América Latina, bem como o sul dos Estados Unidos, continuam sendo regiões endêmicas da DC, mesmo com interferências bem-sucedidas realizadas nesses países quanto à prevenção da doença. O que se observa é que, apesar dos altos gastos realizados no tratamento da doença, já que ela causa, além de cardiopatias graves, doenças digestórias na fase crônica, bem como afastamento do trabalho e custos previdenciários, fica evidente que o esforço maior para o controle e prevenção não são dos governantes, mas sim, dos cientistas que buscam ativamente junto a esses garantir investimentos para as pesquisas com relação a doençą ${ }^{(3)}$.

A DC possui um elevado impacto social, relacionando-a as doenças infecciosas na América Latina onde vários medicamentos foram testado para o seu tratamento, desde a sua descoberta em 1909. Entre estes estão: Bismuto, anti-his- 
tamínicos, Fucsina, Anfotericina B, antibióticos, arsênicos, entre outros, porém o tratamento ainda apresenta algumas controversas. A cura, por sua vez, ainda é muito limitante devido não ter na fase crônica uma terapia com eficácia e devido à ausência de um marcador que defina a cura da $\mathrm{DC}^{(13,21)}$.

De acordo com o Centro de Controle e Prevenção de Doenças, além da pouca efetividade do nifurtimox na fase crônica, esses medicamentos existentes ainda causam efeitos colaterais nos pacientes como dor abdominal, perda de peso, vômitos, dermatites alérgicas, de modo a causar muito desconforto durante o tratamento. É importante evidenciar também que, além dos diferentes perfis de morbimortalidade, apresenta-se onerosa para os cofres públicos em decorrência das inúmeras internações, tendo em vista toda a sintomatologia clássica, sendo ela a cardiomiopatia chagásica, megaesôfago e megacólon; transplantes; absenteísmo, ou seja, a ausência do profissional no trabalho decorrente do excesso de atestados médicos para tratamento da doença; aposentadorias; e óbitos precoces ${ }^{(22)}$.

Além da escassez de medicamentos, fatores como a subnotificação da doença traz prejuízos para a realização de um diagnóstico precoce, bem como para um tratamento mais efetivo na fase aguda da DC. Tendo ainda a falta de testes rápidos para pacientes de regiões endêmicas da doença, falta de triagem para diagnóstico durante o pré-natal ${ }^{(1,23)}$.

Investimentos para realização de novas pesquisas para tratamento da DC humana

Sabe-se que os investimentos realizados sobre novas pesquisas para o tratamento da DC é ainda insuficiente. Entre 1976 e 2014, apenas 117 patentes foram depositadas, tendo um maior deposito no ano de 2019 com nove patentes, o que demonstra que não houve muita evolução no tratamento desde a descoberta da doença. Existem hoje algumas pesquisas sendo realizadas sobre fármacos, vacinas, testes para diagnósti-
De acordo com o Centro de Controle e Prevenção de Doenças, além da pouca efetividade do nifurtimox na fase crônica, esses medicamentos existentes ainda causam efeitos colaterais nos pacientes como dor abdominal, perda de peso, vômitos, dermatites alérgicas, de modo a causar muito desconforto durante o tratamento. cos no pré-natal, mas nada bom o suficiente que pudesse ser liberado para uso em seres humanos, observando a grande falta de interesse das indústrias farmacêuticas e governantes ${ }^{(13)}$.

Pesquisas com fármacos antiparasitários é uma nova proposta que está sendo estudada, sendo uma possível alternativa para o tratamento. Outra opção são os estudos realizados para a criação de uma vacina, a qual teve seu estudo iniciado por Brump e de lá pra cá vive um desencontro constante no labirinto da fatídica "immunité partielle", isso devido às muitas especificidades que a vacina possui, não tendo ainda nenhum resultado positivo que viabilizasse testes em humanos, mas esse problema não apresenta obstáculos impossíveis para a ciência, onde nos últimos 20 anos pesquisas estão sendo realizadas, buscando desenvolver uma vacina de ácido desoxirribonucléico (DNA), a qual vem apresentando resultados positivos com relação à imunidade protetora do antígeno, bem como sob a gravidade da $\mathrm{DC}^{(13,24)}$.

Outra opção de tratamento estudada por Hoft e colaboradores é a proteção da mucosa, já que a maior transmissão nos dias atuais se dar pela via oral, o qual busca a indução de imunidade mucosa contra antígenos de T. cruzi ${ }^{(25)}$.

\section{CONCLUSÃO}

Os estudos científicos encontrados abordam que a DC proporciona ao portador uma condição complexa repercutindo no mau funcionamento sensório-motor, bem como compromete a dimensão biofisiopsicológica, afetando negativamente os aspectos socioeconômicos, embora seja tratável, porém de diagnóstico tardio. Este estudo pôde evidenciar que a DC é negligenciada pelos governantes, não sendo de interesse das grandes indústrias farmacêuticas, tendo em vista que sua prevalência de acometimento é na população economicamente vulneral, de modo a não poder arcar com tratamentos caros. Infere-se que esse desin- 
teresse perdura a muitos anos, refletindo na piora crescente da QV dos portadores de DC. Ainda, muitos trabalhos científi- cos são realizados, mas na sua maioria não evidencia a necessidade na melhora do tratamento da DC, pensando em uma melhor QV dos portadores, já que não conseguem realizar com precisão um diagnóstico precoce da doença.

\section{Referências}

1. Brasil. Ministério da Saúde. Fundação Oswaldo Cruz. Doença de Chagas. Rio de Janeiro: FIOCRUZ, 2013 [cited 2019 jun 19]. Available from: https://agencia.fiocruz. br/doen\%C3\%A7a-de-chagas

2. Diez M, Favaloro L, Bertolotti A, Burgos JM, Vigliano C, Lastra MP et al. Usefulness of PCR strategies for early diagnosis of Chagas' disease reactivation and treatment follow-up in heart transplantation. Am. J. Transplant. [Internet]. 2007 [cited 2020 jul 20];7(6):1633-40. doi: $h$ ttp://dx.doi.org/10.1111/j.1600-6143.2007.01820.x

3. Dias JCP, Ramos Junior AN, Gontijo ED, Luquetti A, Shikanai-Yasuda MA, Coura JR et al. Il Consenso Brasileiro em Doença de Chagas, 2015. Epidemiol. Serv. Saúde [Internet]. 2016 [cited 2020 jun 18];25(esp):7-86. doi: http://dx.doi.org/10.5123/ S1679-49742016000500002

4. Alves DF, Muniz ASC, Abrel CDR, Freitas NR, Teixeira AB, Ferreira ES. Métodos de diagnóstico para a Doença de Chagas: uma atualização. Rev. Bras. An. Clin. [Internet]. 2018 [cited 2020 jun 18];50(4):330-3. doi: http://dx.doi.org/10.21877/24483877.201800726

5. Brasil RGA, Silva PLN, Fonseca JR, Maciel APF, Gonçalves RPF, Souto SGT et al. Análise da soroprevalência da Doença de Chagas em uma cidade do norte de Minas Gerais durante o período de 2012 a 2014. Rev. Univ. Vale Rio Verde [Internet]. 2014 [cited 2020 Jun 18];12(2):734-42. doi: http://dx.doi.org/10.5892/ruvrd.v12i2.1601

6. Forattini OP. Biogeografia, origem e distribuição da domiciliação de triatomíneos no Brasil. Rev. Saúde Pública [Internet]. 2006 [cited 2020 Jul 18];40(6):964-98. https://doi.org/10.1590/S0034-89102006000700004

7. Villar JC, Herrera VM, Carreño JGP, Herrera EV, Dominguez YZC, Vásquez SM et al. Nifurtimox versus benznidazole or placebo for asymptomatic Trypanosoma cruzi infection (Equivalence of Usual Interventions for Trypanosomiasis - EQUITY): study protocol for a randomised controlled trial. BMC [Internet]. 2019 [cited 2020 Jul 18];20(431):1-10. doi: http://dx.doi.org/ 10.1186/s13063-019-3423-3

8. Kropf SP, Azevedo N, Ferreira LO. Doença de Chagas: a construção de um fato científico e de um problema de saúde pública no Brasil. Ciênc. Saúde Colet. [Internet]. 2000 [cited $2020 \mathrm{Jul}$ 18];5(2):347-65. doi: https://doi.org/10.1590/S141381232000000200009

9. Schofield CJ, Jannin J, Salvatella R. The future of Chagas disease control. Trends Parasitol. [Internet]. 2006 [cited 2020 Jul 18];22(12):583-8. doi: http://doi.org/10.1016/j. pt.2006.09.011

10. Petherick A. Chagas disease. Nature (Lond.) [Internet]. 2010 [cited 2020 Jul 18];465(7301):10-1.

11. Coura JR, Castro SL. A critical review on Chagas disease chemotherapy. Mem. Inst. Oswaldo Cruz [Internet]. 2002 [cited 2020 Jul 19];97(1):3-24. doi: https://doi. org/10.1590/S0074-02762002000100001

12. Morilla MJ, Romero EL. Nanomedicines against Chagas disease: an update on therapeutics, prophylaxis and diagnosis. Nanomed. (Lond) [Internet]. 2015 [cited 2020 Jul 19];10(3):465-81. doi: https://doi.org/10.2217/nnm.14.185

13. Cruz CAB, Silva ALS, Alencar EMD, Santos NJB, Moreira JJS, Paixao AEA et al. Tecnologias que empregam fármacos antiparasitários para tratamento da doença Chagas. Rev. Eletr. Comun. Inf. Inov. Saúde [Internet]. 2016 [cited 2020 Jul 18];10(1):1-9: Available from: https://www.reciis.icict.fiocruz.br/index.php/reciis/article/view/1075/pdf_1075 14. Silva ACC. Investigação da atividade antiparasitária e imunomoduladora de uma nova classe de compostos tiazolidinonicos na doença de Chagas [Internet]. Recife. 90 fls. Dissertação (Mestrado em Biociências e Biotecnologia em Saúde) - Instituto Aggeu Magalhães, Fundação Oswaldo Cruz, 2018 [cited 2020 Jun 18]. Available from: https://www.arca.fiocruz.br/handle/icict/27528

15. Vinhaes MC, Dias JCP. Doença de Chagas no Brasil. Cad. Saúde Pública [Internet]. 2000 [cited 2020 May 27];16(Suppl 2):S7-S12. doi: https://doi.org/10.1590/S0102$311 \times 2000000800002$

16. Coura JR, Dias JCP. Epidemiologia, controle e vigilância da doença de Chagas: 100 anos após sua descoberta. Mem. Inst. Oswaldo Cruz [Internet]. 2009 [cited 2020 Jul 18];104(Supl. 1):31-40. doi: https://doi.org/10.1590/S0074-02762009000900006 17. Dias JCP, Vinhaes MC, Silveira AC, Schofield CJ, Cardoso B, Coura JR. Pesquisas prioritárias sobre doença de Chagas na Amazonia: agenda de curto-médio prazo. Rev. Soc. Bras. Med. Trop. [Internet]. 2001 [cited 2020 Jul 18];34(5):497-8. doi: http:// doi.org/10.1590/S0037-86822001000500017

18. Victora CG, Wagstaff A, Schellenberg JA, Gwatkin D, Claeson M, Habicht J. Applying an equity lens to child health and mortality: more of the same is not enough. Lancet [Internet]. 2003 [cited 2020 Jul 18];362(9379):233-41. doi: http://doi. org/10.1016/50140-6736(03)13917-7

19. Dias JCP, Cláudio LDG, Lima MM, Albajar-Viñas P, Silva RA, Alves RV et al. Mudanças no paradigma da conduta clínica e terapêutica da doença de Chagas: avanços e perspectivas na busca da integralidade da saúde. Epidemiol. Serv. Saúde [Internet]. 2016 [cited 2020 Jul 18];25(esp):87-90. doi: http://doi.org/10.5123/S167949742016000500003

20. Lima RS, Teixeira AB, Lima VLS. Doença de Chagas: uma atualização bibliográfica. Rev. Bras. Anal. Clín. [Internet]. 2019 [cited 2020 Jul 20];51(2):103-6. doi: http://doi. org/10.21877/2448-3877.201900727

21. Lima RS, Soares MBP, Santos RR. Terapia celular na doença de Chagas. Rev. Bras Hematol. Hemoter. [Internet]. 2009 [citado 2020 May 28];31(Supl. 1):87-92. doi: https://doi.org/10.1590/S1516-84842009005000037

22. Ramos Junior AN, Carvalho DM. Chagas' disease: past, present and future [Editorial]. Cad. Saúde Colet. [Internet]. 2009 [cited 2020 May 28];17(4):787-94. Available from: https://www.researchgate.net/publication/247768733_Editorial_-_Chagas'_ Disease_past_present_and_future

23. Brasil. Ministério da Saúde. Secretaria de Vigilância em Saúde. Doença de Chagas aguda no Brasil: série histórica de 2000 a 2013. Bol. Epidemiol. [Internet]. 2015 [cited 2020 Jun 18];46(21):1-9. Available from: http://portalarquivos.saude.gov.br/images/ pdf/2015/agosto/03/2014-020..pdf

24. Garg N, Tarleton RL. Genetic immunization elicits antigen-specific protective immune responses and decreases disease severity in Trypanosoma cruzi infection. Infect. Immun. [Internet]. 2002 [cited 2020 Jul 18];70(10):5547-55. doi: http://doi. org/10.1128/IAl.70.10.5547-5555.2002

25. Hoft DF, Eickhoff CS. Type 1 immunity provides both optimal mucosal and systemic protection against a mucosally invasive, intracellular pathogen. Infect. Immun. [Internet]. 2005 [cited 2020 Jul 18];73(8):4934-40. doi: http://doi.org/10.1128/ IAl.73.8.4934-4940.2005

26. Passos LCS, Melo RMV, Lira YM, Oliveira NFC, Trindade T, Carvalho W et al. A doença de Chagas está associada a um desfecho ruim no acompanhamento de 1 ano após a terapia de ressincronização cardíaca. Rev. Assoc. Med. Bras. [Internet]. 2019 [cited 2020 Jul 19];65(11):1391-6. doi: https://doi.org/10.1590/18069282.65.11.1391

27. Monteiro JMC, San-Martin DL, Silva BCG, Jesus PAP, Oliveira Filho J. Anticoagulação em pacientes com manifestações cardíacas da doença de Chagas e acidente vascular cerebral isquêmico cardioembólico. Arq. Neuro-Psiquiatr. [Internet]. 2018 [cited 2020 Ago 1];76(1):22-5. doi: https://doi.org/10.1590/0004-282×20170180 28. Leite ACA, Moreira MAR, Barbosa MA, Moreira Júnior H, Leite PCCA, Moreira JPT. Investigação clínica e manométrica em chagásicos constipados com e sem megacólon. J. Coloproctol. (Rio J.) [Internet]. 2019 [cited 2020 Ago 2];39(2):145-52. doi: https://doi.org/10.1016/j.jcol.2018.12.002

29. Rassi DC, Vieira MLC, Furtado RG, Turco FP, Melato LH, HottaVT et al. Segurança do Ecocardiograma sob Estresse com Dobutamina-Atropina em Pacientes com Doença de Chagas. Arq. Bras. Cardiol. [Internet]. 2017 [cited 2020 Ago 27];108(2):122-8. doi: https://doi.org/10.5935/abc.20170002

30. Coura JR, Borges-Pereira J. Chagas disease: What is known and what should be improved: a systemic review. Rev. Soc. Bras. Med. Trop. [Internet]. 2012 [cited 2020 Ago 27];45(3):286-96. doi: https://doi.org/10.1590/S0037-86822012000300002 31. Barbosa-Ferreira JM, Guerra JAO, Santana Filho FS, Magalhães BML, Coelho LIARC, Barbosa MGV. Acometimento cardíaco em casos de doença de Chagas aguda da Amazônia. Arq. Bras. Cardiol. [Internet]. 2010 [cited 2020 Ago 27];94(6):147-9. doi: https://doi.org/10.1590/50066-782X2010000600023 